\title{
Enhanced Endothelialization of Dacron Grafts by External Vein Wrapping
}

\author{
Linda M. Graham, M.D., Karyn A. Harrell, B.A., Ruth L. Sell, \\ James W. Crudup, William E. Burkel, Ph.D., and James C. Stanley, M.D. \\ Departments of Surgery and Anatomy and Cell Biology, University of Michigan Medical School, \\ Ann Arbor, Michigan 48109
}

Presented at the Annual Meeting of the Association for Academic Surgery, San Antonio, Texas, October 31-November 3, 1984

\begin{abstract}
The efficacy of external vein wrapping of vascular grafts in promoting the development of a luminal endothelial surface was assessed in 12 dogs who underwent thoracoabdominal bypasses with 26-29 $\mathrm{cm} \times 6-\mathrm{mm}$ i.d. double-velour knitted Dacron grafts. In group I $(n=6) 6-\mathrm{cm}$ segments of the grafts were wrapped with autologous jugular vein with its endothelial surface applied against the outside of the graft. In group II $(n=6)$ the wrap procedure was performed using abdominal wall fascia. The degree and character of graft incorporation was quantitated in all prostheses at 28 days postimplantation. Group I vein wrap prostheses demonstrated uniform endothelial surface coverage in the vein wrap area $(\bar{x}=88 \%)$ that was significantly greater $(P<0.04)$ than coverage in adjacent unwrapped segments $(\bar{x}=48 \%)$. Group II fascial wrap grafts demonstrated variable endothelial coverage in the wrapped area $(\bar{x}=48 \%)$ that was consistently less than in adjacent unwrapped portions $(\bar{x}=62 \%)$, and significantly less than vein wrap sections of group I animals $(P<0.04)$. No significant differences existed in endothelial coverage of unwrapped regions of group I and II grafts. This investigation documented that wrapping knitted Dacron grafts with vein enhanced endothelialization of their luminal surface. (1) 1985 Academic Press, Inc.
\end{abstract}

\section{INTRODUCTION}

Early development of an endothelial cell luminal surface has been shown in laboratory studies to improve patency of small caliber protheses $[1-3,11,13,15]$. Unfortunately, vascular grafts used in humans rarely exhibit endothelium beyond the few centimeters of anastomotic pannus ingrowth [14]. Endothelial cell seeding has been proposed as one means of facilitating development of an antithrombogenic surface of endothelium on grafts [1-5, 7-11, 15]. Rosenfeld and his coworkers suggested an alternative method based on a canine model in which short carotid artery grafts, wrapped with a vein on their external surface, developed an endothelial lining [13]. The objective of the present investigation was to assess the efficacy of wrapping central segments of long, knitted Dacron grafts with autologous vein to produce a luminal endothelial surface.

\section{MATERIALS AND METHODS}

Twelve adult mongrel dogs weighing 18 to $35 \mathrm{~kg}$ underwent thoracoabdominal bypasses with $6 \mathrm{~mm}$ i.d., 26 to $29 \mathrm{~cm}$ long, knitted double-velour Dacron grafts. Animals were randomized into two groups (I and II) for study. In group I $(n=6)$ a segment of autologous jugular vein was removed, slit, and applied with its endothelial surface against a midsection of the graft (Fig. 1). In group II $(n=6)$ a similar wrap procedure was carried out using the external abdominal oblique aponeurosis (fascia) instead of jugular vein. Animal husbandry was provided by the staff of the University of Michigan Unit for Laboratory Animal Medicine which is fully accredited by the American Association for Accreditation of Laboratory Animal Care. Surgical procedures and animal care complied with the standards in "The Guide for the Care and Use of Laboratory Animals" 


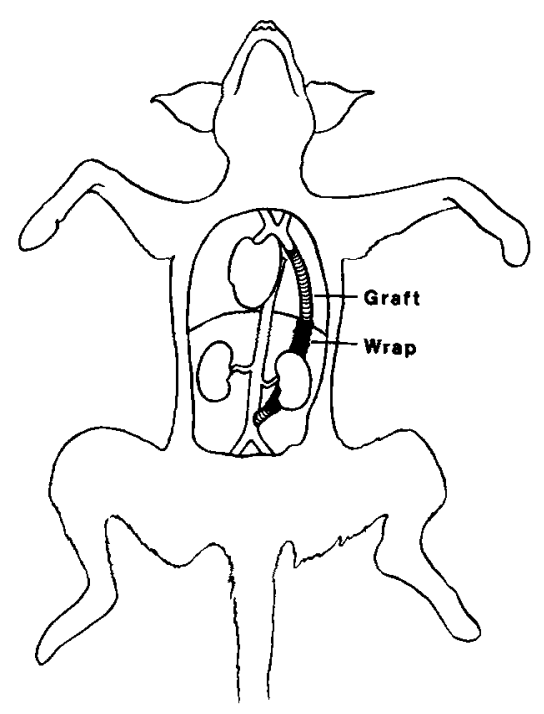

FIG. 1. Diagram of model showing thoracoabdominal bypass graft with the wrap on its external surface.

[DHEW Publication No. (NIH) 78-23, revised 1978].

Double-velour, warp-knitted Dacron prostheses were used for the bypass grafts. These conduits were characterized by an inside pile of $0.15 \mathrm{~mm}$, outside pile of 0.29 $\mathrm{mm}$, water porosity of $1300 \pm 400 \mathrm{ml} / \mathrm{min} /$ $\mathrm{cm}^{2}$, interstices of greater then $3000 / 2.5 \mathrm{~cm}^{2}$, and pore size of 2 to $400 \mu \mathrm{m}$. Grafts were preclotted with $30 \mathrm{ml}$ autologous blood flushed through the conduit until thrombus occluded the interstices. The prostheses were then rinsed with $15 \mathrm{ml}$ of autologous blood containing 5000 units of heparin sodium.

In preparation for thoracoabdominal bypass graft placement, dogs were anesthetized with $30 \mathrm{mg} / \mathrm{kg}$ of pentobarbital sodium, intubated, and mechanically ventilated. All animals were hydrated during the procedure with $15 \mathrm{ml} / \mathrm{kg} / \mathrm{hr}$ of lactated Ringer's solution. The graft was first anastomosed end-to-side to the infrarenal aorta using a continuous 5O polypropylene suture. Following administration of heparin sodium (100 units $/ \mathrm{kg}$ ), the thoracic aorta distal to the left subclavian artery was clamped and divided. An end-toend anastomosis between the graft and the proximal descending thoracic aorta was then performed with a continuous 5-O polypro- pylene suture. The distal thoracic aorta was oversewn. After removal of all clamps, the heparin's anticoagulant effect was reversed with protamine sulfate $(1.0 \mathrm{mg} / \mathrm{kg})$. Penicillin $\mathrm{G}$ benzathine and procaine hydrochloride (450,000 units each) were administered to all animals preoperatively.

In group I dogs, an external jugular vein was removed and opened longitudinally. A 6-cm intraabdominal portion of the previously placed graft was wrapped with this vein segment with its endothelial surface against the external surface of the prosthesis. In group II dogs, a portion of external abdominal oblique aponeurosis was removed and wrapped in a similar fashion around a $6-\mathrm{cm}$ segment of the graft at least $2.5 \mathrm{~cm}$ above the distal anastomosis.

All prostheses were removed and examined 28 days postimplantation. Following careful dissection of the graft and adjacent vessels, dogs were anticoagulated with heparin sodium (150 units $/ \mathrm{kg}$ ), and perfusion cannulas were inserted into the left subclavian artery and distal infrarenal aorta. Clamps were then applied to the aorta proximal to the cannulated left subclavian artery. and distal to the graft. The isolated prosthesis was flushed with Dulbecco's phosphate-buffered saline. Following this, the graft was perfusion fixed with $4 \%$ paraformaldehyde and removed. The prosthesis was then divided longitudinally and 35-mm color photographic transparencies were taken of the inner graft surface. Onehalf of the longitudinally transected graft was cut into random sections of anastomotic regions, as well as wrapped and unwrapped regions, for study by light microscopy. These sections were fixed in $2.5 \%$ glutaraldehyde, embedded in glycol methacrylate, and stained with methylene blue-basic fuchsin. The other half of the graft was divided into 0.5 - to 1 cm-long segments, fixed in $2.5 \%$ glutaraldehyde, dehydrated in ethanol, and critical point dried for scanning electron microscopy.

Inner capsule tissue characteristics and thicknesses were evaluated by light microscopy. For consistency the thickness of the inner capsule was measured at the top or 
peak of the Dacron fiber ridges. Using a dissecting microscope and scanning electron microscopy for confirmation, the percentage of endothelial cell surface coverage was determined on specimens composing one longitudinal half of the 6-cm wrapped segment of each graft, as well as 10 random specimens from the remainder of each graft. The endothelial coverage of each specimen was evaluated by two independent observers. The coverage was considered $0 \%$ if no endothelium was seen or $100 \%$ if the specimen was covered completely with endothelium. When partial coverage was observed, a grid was utilized as necessary. The presence or absence of endothelium at 10 points of intersection was evaluated to arrive at an estimate of percentage endothelial coverage. Statistical comparisons of inner capsule thicknesses and of endothelial coverage were made using analysis of variance and Student's $t$ test.

\section{RESULTS}

All prostheses were patent at the time of their removal 28 days postimplantation. Vein wrapped areas of group I grafts exhibited a smooth, glistening, transparent lining with little red thrombus. In contrast, unwrapped areas of group I grafts were red and mottled in appearance. Fascial wrapped portions of group II grafts were not different in gross appearance from the unwrapped segments of these grafts, exhibiting red clot in some portions while other areas had little thrombus.
Outer capsules in wrapped areas of both group I and II grafts were grossly thicker than in unwrapped areas.

Light microscopy of group I vein wrap graft segments demonstrated a thin inner capsule surfaced by endothelium (Fig. 2). In some regions endothelial cells were in direct juxtaposition to the graft fibers. More commonly a few layers of smooth muscle cells were interposed between the Dacron fibers and the surface endothelium. Between graft fiber ridges, fibroblasts and collagenous material could be identified beneath the smooth muscle. Graft interstices in wrapped areas were completely incorporated, with giant cells and macrophages predominating in this tissue. No fibrin remained, and vasa vasorum were frequently identified within the interstices and deeper parts of the inner capsule of the graft. The outer capsule of group I vein wrapped segments was thicker than the outer capsule of unwrapped segments. In the wrapped segments a band of four to six layers of smooth muscle cells, elastic fibers, and collagen, representing the vein wrap itself, was evident. In some regions a dense network of relatively large blood vessels was present between the vein remnant and the outer graft fibers (Fig. 2).

Inner capsules of group II fascial wrapped graft segments demonstrated an unstable fibrin-platelet coagulum of variable thickness that extended into the interstices of the graft itself (Fig. 3A). Occasional segments had a

TABLE I

\begin{tabular}{|c|c|c|c|c|c|}
\hline & $\begin{array}{c}\text { Inner capsule } \\
\text { thickness } \\
(\mu \mathrm{m}) \\
(x \pm \mathrm{SD})\end{array}$ & & $\begin{array}{c}\text { Endothelial } \\
\text { coverage } \\
(\%) \\
(x \pm \mathrm{SD})\end{array}$ & & \\
\hline \multicolumn{6}{|l|}{ Group I (vein) } \\
\hline Wrapped & $21.7 \pm 16.1$ & & $88 \pm 16$ & \multirow{3}{*}{$P<0.04$} & \multirow{6}{*}{$P<0.04$} \\
\hline \multirow[t]{2}{*}{ Unwrapped } & $42.2 \pm 37.3$ & & $48 \pm 10\}$ & & \\
\hline & & $P<0.02$ & & & \\
\hline \multicolumn{5}{|c|}{ Group II (fascia) } & \\
\hline Wrapped & $77.5 \pm 38.1$ & & $48 \pm 10$ & & \\
\hline Unwrapped & $72.7 \pm 52.6$ & & $62 \pm 30$ & & \\
\hline
\end{tabular}




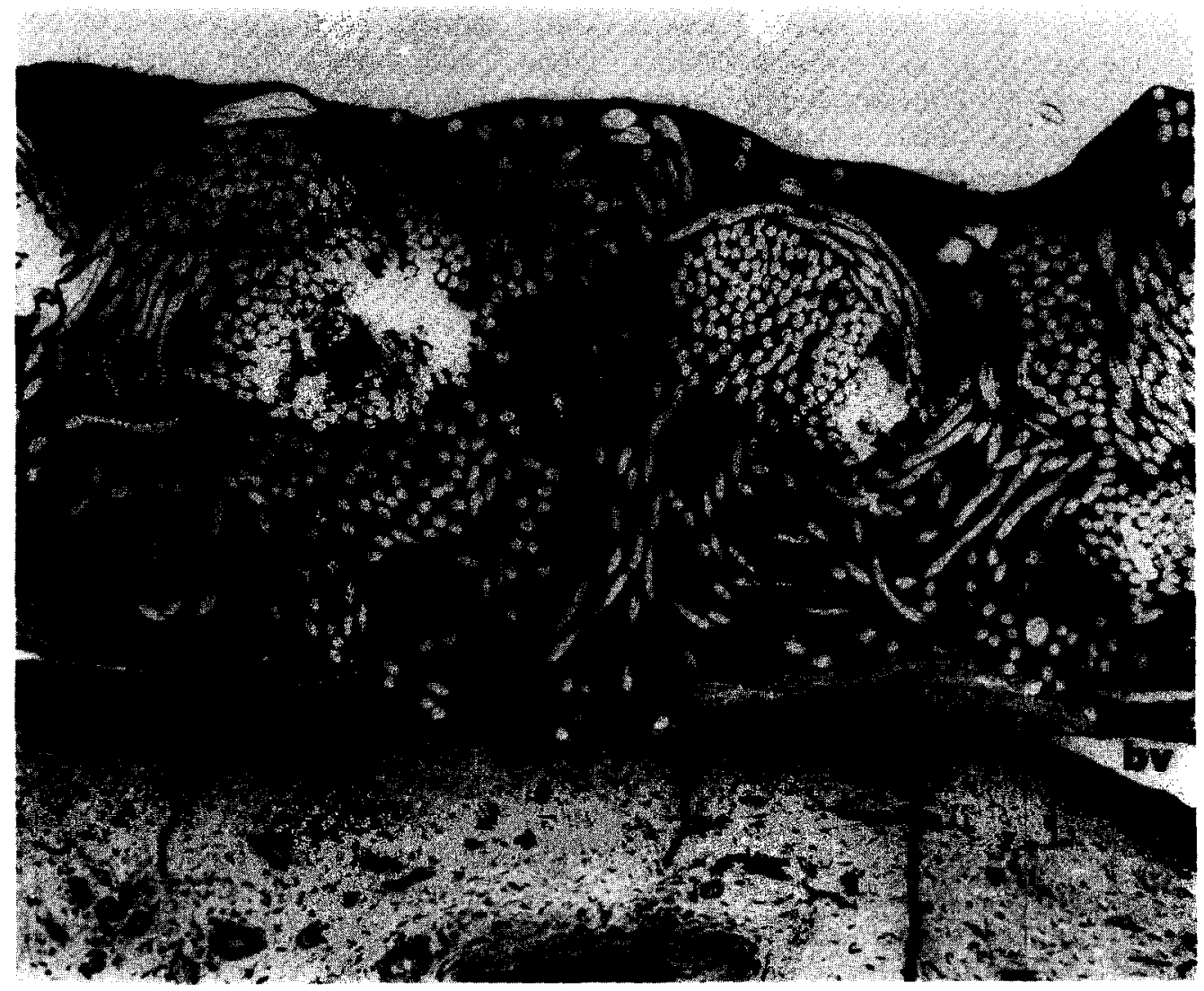

FIG. 2. Low-power light micrograph of graft segment wrapped with external jugular vein. In its thickest regions the inner capsule is composed of collagen, fibroblasts, vasa vasora, and a few layers of sinooth muscle cells surfaced by a layer of endothelium. In many areas only a single layer of endothelial cells separates the graft fibers from the blood. The interstices contain predominantly macrophages and foreign body giant cells. At the bottom of the field, the multiple layers of smooth muscle cells (sm) and elastic fibers from the vein wrap can be identified. Interposed between the vein and the graft, a large blood vessel is seen (bv). Methylene blue and basic fuchsin, $\times 100$.

thinner inner capsule with a surface of endothelium (Fig. 3B). Healing of the fascial wrapped area was usually less advanced than the adjacent unwrapped areas. Cellular ingrowth in most wrapped areas was restricted to peripheral interstitial regions of the graft and consisted mainly of giant cells and macrophages. The outer capsule of group II fascial wrapped graft segments was similar to that of the unwrapped regions with the exception of dense collagenous bundles of the aponeurosis in the former. The outer capsule in the area of the fascial wrap was also generally thicker than in adjacent unwrapped areas.

Unwrapped portions of group I and II grafts were similar. Inner capsules were composed of multilayered coagulum of fibrin with platelets and trapped blood cells in most regions (Fig. 4). However, areas were identified in which the inner capsule was thinner and surfaced by endothelium. The outer capsule of unwrapped portions of grafts was composed of collagenous tissue with giant cells and macrophages infiltrating interstices of the graft.

Inner capsule thickness of group I vein wrapped segments was $21.7 \pm 16.1 \mu \mathrm{m}$ $(\bar{x} \pm \mathrm{SD})$ (Table I). This was significantly less than the $77.5 \pm 38.1 \mu \mathrm{m}$ inner capsule thickness of fascial wrapped segments in 


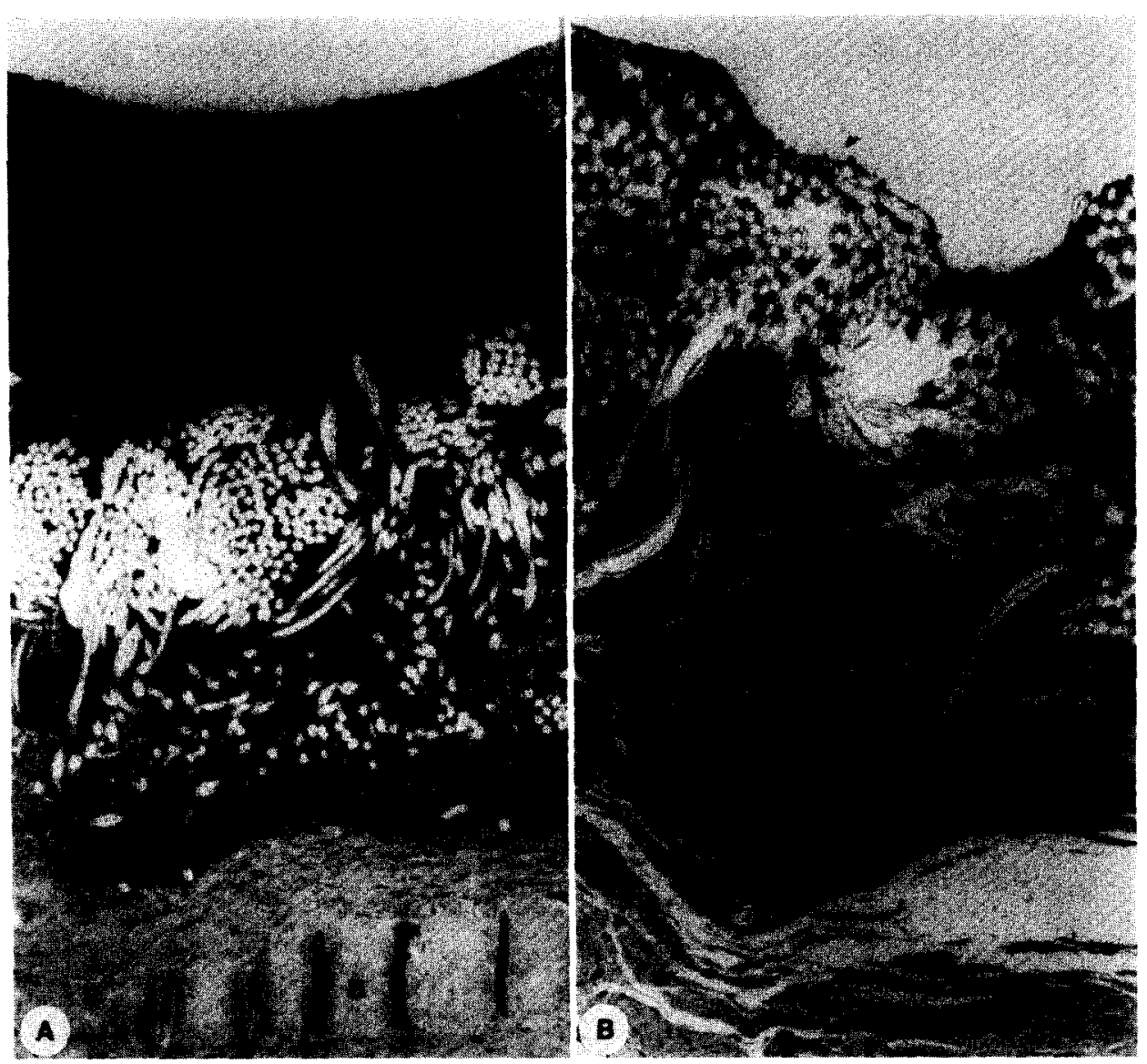

FIG. 3. (A) Photomicrograph of fascial-wrapped portion of a group II graft. The inner capsule of the graft is composed of a thick, friable, poorly organized fibrin-platelet coagulum. The material filling the interstices of the inner half of the graft is acellular, being primarily fibrin. In the interstices of the outermost portion of the graft, the tissue is better organized with giant cells and macrophages present. In the outer capsule the dense, relatively acellular, avascular aponeurosis (a) of the external abdominal oblique muscle used to wrap the graft is clearly evident. Vasa vasora are not present in the graft. Methylene blue and basic fuchsin, $\times 120$. (B) Light micrograph of another section through the fascialwrapped portion of a group II graft. The outer capsule and interstices are typical. The inner capsule is extremely thin and a monolayer of endothelium is clearly evident on the surface. By 1 month endothelialized regions are occasionally seen in porous grafts in the dog model. Methylene blue and basic fuchsin, $\times 110$.

group II dogs $(P<0.02)$. Inner capsule thickness in the unwrapped thoracic segments of the group I grafts averaged $42.4 \pm 37.3$ $\mu \mathrm{m}$ which was not significantly different $(P$ $=0.4$ ) from the aforementioned thickness of the wrapped segments. The inner capsule thicknesses in group II wrapped $(77.5 \pm 38.1$ $\mu \mathrm{m})$ and unwrapped $(72.7 \pm 52.6 \mu \mathrm{m})$ areas were not significantly different. Similarly, the inner capsule thicknesses of unwrapped thoracic graft segments of group I (42.2 \pm 37.3 $\mu \mathrm{m})$ and of group II $(72.7 \pm 52.6 \mu \mathrm{m})$ were not significantly different.

Luminal surfaces of group I vein wrapped graft segments demonstrated a confluent cellular surface with very few adherent platelets (Fig. 5A), The surface was typical of endothelium with nonoverlapping intercellular junctions and prominent nuclear bulges. These squamous cells were oriented with their long axis in the direction of blood flow. Endothelium covered $88 \pm 16 \%(\bar{x} \pm \mathrm{SD})$ of 


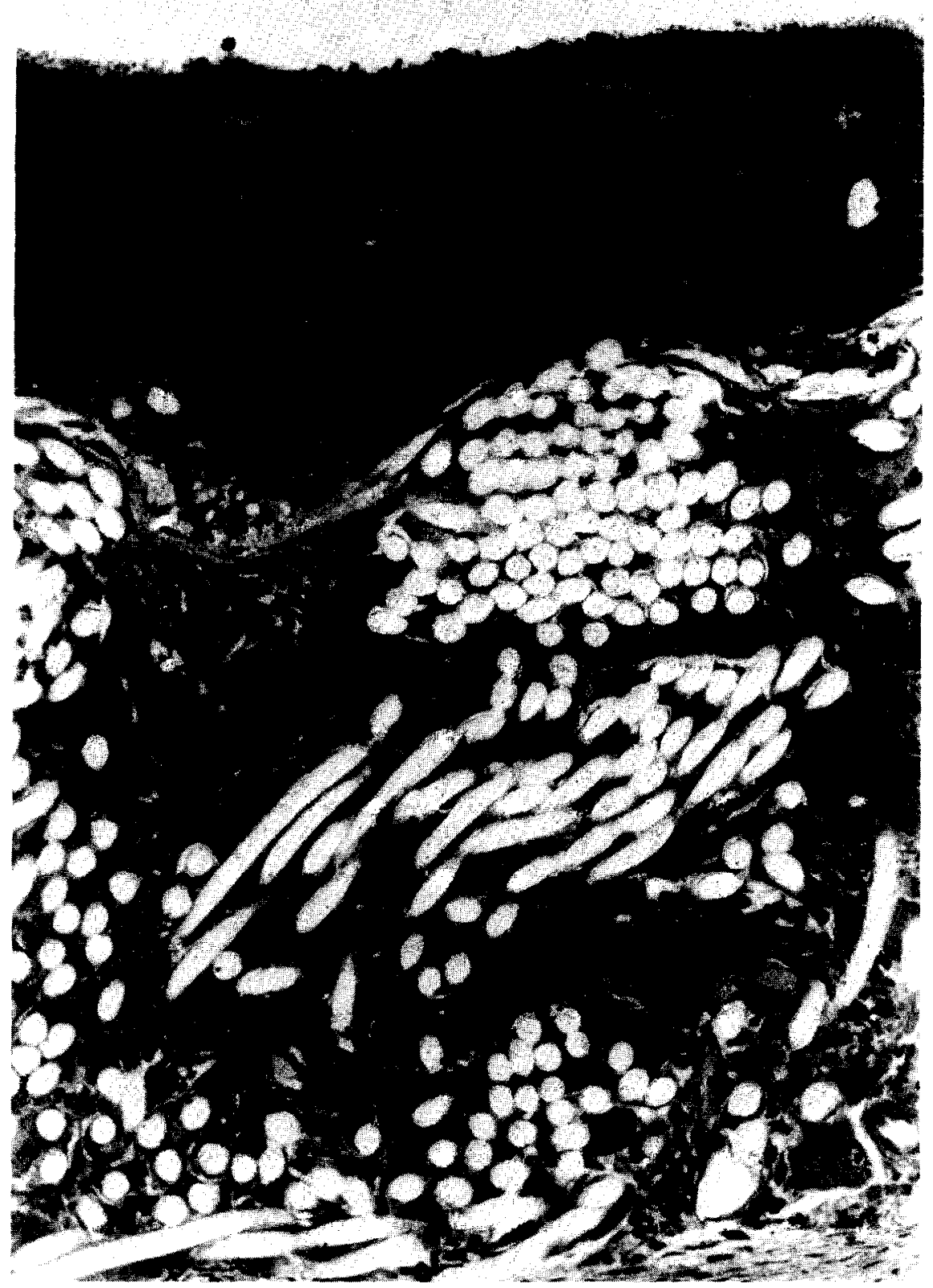

FIG. 4. Light micrograph of the unwrapped portion of a group I graft. This section displays the characteristic healing pattern of an unseeded, unwrapped graft in the dog model. The outer capsule, lower right, is composed of dense collagenous tissue and the interstices are very cellular, being infiltrated with giant cells and macrophages. The deeper part of the inner capsule is made up of fibroblasts and collagen while the luminal portion and surface is comprised of fibrin with adherent white blood cells and platelets. Methylene blue and basic fuchsin, $\times 220$.

the surface in regions of the vein wrap (Table I). Adjacent unwrapped segments of the same group I grafts had consistently less endothelial coverage ( $48 \pm 10 \%)$. These differences were statistically significant $(P<0.04)$. Unwrapped portions demonstrated large surface areas 


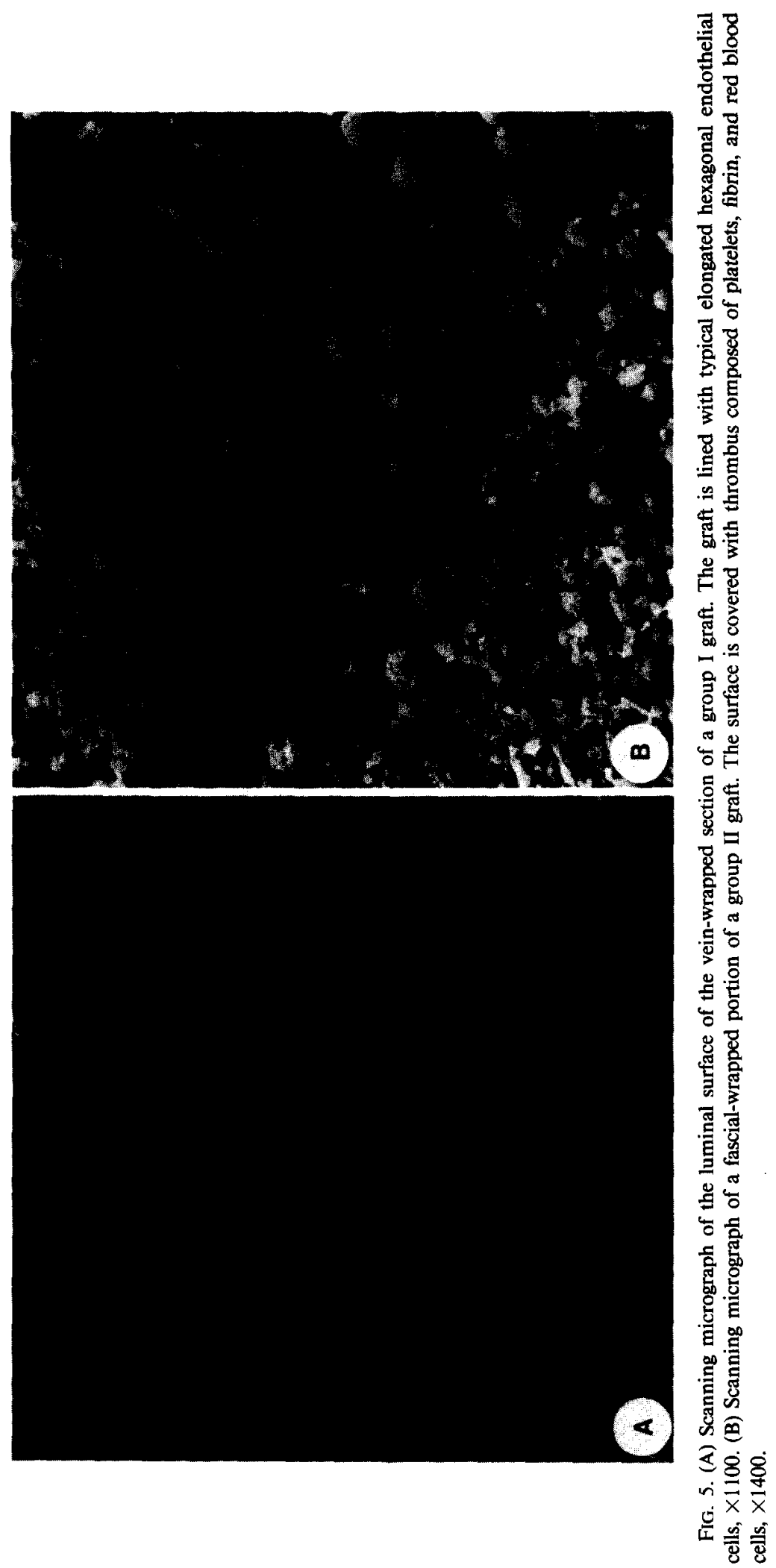


covered with fibrin with adherent platelets and blood cells. Similarly, group II fascial wrapped graft segments exhibited large areas of thrombus on their luminal surface (Fig. 5B). Endothelial coverage of fascial wrapped portions $(48 \pm 10 \%)$ was consistently less than adjacent unwrapped sections (62 $\pm 30 \%$ ), but this difference was not statistically significant. Endothelial coverage in the group $I$ vein wrapped segments was significantly greater than in the group II fascial wrapped segments $(P<0.04)$. Significant differences in endothelial coverage of unwrapped portions of group I and II grafts were nonexistent.

\section{DISCUSSION}

A recent study by Rosenfeld and his coworkers suggested that endothelial cells from a slit autologous vein wrapped about the external surface of a 2.5 - to $4.0-\mathrm{cm}$-long knitted Dacron carotid artery graft promoted early development of an endothelial lining [13]. Of their wrapped grafts 18 of 20 were patent for study periods up to 250 days. All patent grafts had scanning electron microscopic evidence of cellular linings characteristic of endothelium. In this same experiment only 11 of 20 unwrapped interposition carotid artery grafts remained patent during a similar study period. Surprisingly, none of these unwrapped grafts exhibited a cellular lining. This was in distinct contrast to our experience where an endothelial lining has been noted in some nonpannus areas of knitted Dacron grafts 4 to 8 weeks after implantation [4].

The present investigation was undertaken to better define the role of vein wrapping in the promotion of endothelial surfacing in knitted Dacron prostheses. A thoracoabdominal graft model was chosen to allow comparison between a wrapped segment and an adjacent unwrapped segment of prosthesis. Furthermore, since previous studies from our laboratory had documented anastomotic pannus ingrowth in this long graft to be less than $1 \mathrm{~cm}$ at 4 weeks postimplantation, nonpannus endothelial cell coverage could be distinguished from pannus ingrowth $[7,8]$.
This study also addressed the role of the type of wrap in promoting graft endothelialization. The external abdominal oblique aponeurosis was selected as the "control tissue" because of its relatively avascular nature. Although the fascia could not be completely cleared of investing tissue, the paucity of capillaries, and thus endothelial cells, on the fascia was in distinct contrast to the large number of endothelial cells on the surface of the slit vein. The more rapid development of an endothelial surface in the vein wrapped segment might be attributed to a direct effect of the presence of endothelium in the tissue used to wrap the graft. One possible explanation for our results is that endothelial cells originating from the intima of the vein migrated through the interstices of the graft.

Earlier experiments conducted by others suggested that endothelial cells might migrate through vascular graft interstices and form a true endothelial lining $[6,12]$. In 1962 Florey and colleagues reported the development of an endothelial cell surface on Dacron grafts in part from small vascular channels that appeared to pass through interstices of the graft fabric [6]. Macpherson and Muir identified an endothelial luminal surface in central portions of porous grafts, but not in the midportions of nonporous grafts implanted for 1 year in dogs [12]. These authors concluded that transinterstitial growth of cells was the most important source of surface endothelium in regions distant from anastomotic pannus ingrowth. Since the grafts in the present study were evaluated only at 28 days, no definitive conclusions should be rendered regarding the precise source of the endothelium on the graft's luminal surface.

Data from the present study support the tenet that the development of endothelium on the luminal surface of a porous, prosthetic graft is promoted by vein wrapping. The luminal surface of these vein-wrapped sections showed significantly more endothelial cell coverage than adjacent unwrapped regions or fascia-wrapped graft segments. Indeed, fascia wrapping appeared detrimental to graft healing. The inner capsule of the 
fascia-wrapped portions was composed of poorly organized thrombus in many areas, and was significantly thicker than the inner capsule of vein-wrapped segments. In addition, fascia wrapped graft interstices had less cellular ingrowth than adjacent unwrapped regions or vein-wrapped areas.

\section{REFERENCES}

1. Allen, B. T., Long, J. A., Clark, R. E., Sicard, G. A., Hopkins, K. T., and Welch, M. J. Influence of endothelial cell seeding on platelet deposition and patency in small-diameter Dacron arterial grafts. $J$. Vasc. Surg. 1: 224, 1984.

2. Allen, B. T., Long, J. A., Welch, M. J., Hopkins, K. T., Sicard, G. A., and Clark, R. E. Effect of aspirin therapy and its withdrawal on control and endothelial cell seeded grafts. Surg. Forum 34: 470, 1983.

3. Belden, T. A., Schmidt, S. P., Falkow, L. J., and Sharp, W. V. Endothelial cell seeding of smalldiameter vascular grafts. Trans. Amer. Soc. Artif. Intern. Organs 28: 173, 1982.

4. Burkel, W. E., Vinter, D. W., Ford, J. W., Kahn, R. K., Graham, L. M., and Stanley, J. C. Sequential studies of healing in endothelial seeded vascular prostheses. Histologic and ultrastructure characteristics of graft incorporation. J. Surg. Res. 30: 305, 1981.

5. Burkel, W. E., Ford, J. W., Vinter, D. W., Kahn, R. H., Graham, L. M., and Stanley, J. C. Fate of knitted Dacron velour vascular grafts seeded with enzymatically derived autologous canine endothelium. Trans. Amer. Soc. Artif. Intern. Organs 28: 178, 1982.

6. Florey, H. W., Greer, S. J., Kiser, J., Poole, J. C. F., Telander, R., and Werthessen, N. T. The develop- ment of the pseudointima lining fabric grafts of the aorta. Brit. J. Exp. Pathol. 43: 655, 1962.

7. Graham, L. M., Burkel, W. E., Ford, J. W., Vinter, D. W., Kahn, R. H., and Stanley, J. C. Immediate seeding of enzymatically derived autologous canine endothelium in Dacron vascular grafts: Early experimental studies with autologous canine cells. Arch. Surg. 115, 1289, 1980.

8. Graham, L. M., Vinter, D. W., Ford, J. W., Kahn, R. H., Burkel, W. E., and Stanley, J. C. Endothelial cell seeding of prosthetic vascular grafts: Early experimental studies with cultured autologous canine endothelium. Arch. Surg. 115: 929, 1980.

9. Herring, M. B., Dilley, R., Jersild, R. A., Jr., Boxer, L., Gardner, A., and Glover, J. Seeding arterial prostheses with vascular endothclium: The nature of the lining. Ann. Surg. 190: 84, 1979.

10. Herring, M., Garner, A., and Glover, J. A singlestaged technique for seeding vascular grafts with autogenous endothelium. Surgery 84: 498, 1978.

11. Hunter, T. J., Schmidt, S. P., Sharp, W. V., and Malindzak, G. S. Controlled flow studies in $4 \mathrm{~mm}$ endothelialized Dacron grafts. Trans. Amer. Soc. Artif. Intern. Organs 29: 177, 1983.

12. Macpherson, A. I. S., and Muir, A. R. Synthetic fabric prostheses implanted 1 to 6 years: Studies by light and electron microscopy. Scot. Med. J. 8: 262, 1963.

13. Rosenfeld, J. C., Savarese, R. P., McCombs, P. R., and DeLaurentis, D. A. Endothelial infiltration and lining of knitted Dacron arterial grafts. Surg. Forum 32: 336, 1981.

14. Sauvage, L. R., Berger, K. E., Wood, S. J., Yates, S. G., Smith, J. C., and Mansfield, P. B. Interspecies healing of porous arterial prostheses. Arch. Surg. 109: 698, 1974.

15. Stanley, J. C., Burkel, W. E., Ford, J. W., Vinter, D. W., Kahn, R. H., Whitehouse, W. M., Jr., and Graham, L. M. Enhanced patency of small-diameter, externally supported Dacron iliofemoral grafts seeded with endothelial cells. Surgery 92: 994, 1982. 